\title{
Upregulation of nuclear factor- $\kappa B$ and acid sensing ion channel 3 in dorsal root ganglion following application of nucleus pulposus onto the nerve root in rats
}

\author{
DONG WANG ${ }^{1}$, HAO PAN ${ }^{2}$, HANG ZHU ${ }^{2}$, LI ZHU ${ }^{2}$, YONG-JIANG HE ${ }^{2}$, JIAN WANG ${ }^{2}$ and GAO-YONG JIA ${ }^{2}$ \\ ${ }^{1}$ Department of Orthopaedics, Hangzhou Ding Qiao Hospital, Hangzhou, Zhejiang 310021; ${ }^{2}$ Department of Orthopaedics, \\ The Affiliated Guang-Xing Hospital of Zhejiang TCM University, Hangzhou, Zhejiang 310007, P.R. China
}

Received August 19, 2016; Accepted May 30, 2017

DOI: $10.3892 / \mathrm{mmr} .2017 .7094$

\begin{abstract}
The nucleus pulposus (NP) is an avascular, hydrated tissue that permits the intervertebral disc to resist compressive loads to the spine. To determine the mechanisms by which intervertebral disc degeneration is caused by the nucleus pulposus, the expression and regulation of nuclear factor $(\mathrm{NF})-\kappa \mathrm{B}$ and acid sensing ion channel 3 (ASIC3) were examined. For the intervertebral disc degeneration model, NP was harvested from the tail of rats and applied to the L5 dorsal root ganglion (DRG). The mechanical pain withdrawal threshold (PWT) in NP model rats was assessed. Reverse transcription-quantitative polymerase chain reaction and western blotting were used to examine $\mathrm{NF}-\kappa \mathrm{B}$ and ASIC3 expression levels in DRG. Finally, the effect of the NF- $\kappa \mathrm{B}$ inhibitor pyrrolidine dithiocarbamate (PDTC) and the ASIC3 signaling pathway blocker amiloride were examined. Rats exposed to NP exhibited decreased PWT for 12 days, and NF- $\mathrm{BB}$ and ASIC3 was upregulated in DRG induced by NP 14 days after surgery. After administration of amiloride and PDTC to DRG affected by NP, the levels of nitric oxide (NO), tumor necrosis factor- $\alpha$ (TNF- $\alpha$ ), interleukin-6 (IL-6), NF- $\kappa$ B and ASIC3 were downregulated, and the levels of aquaporin (AQP) 1 and AQP3 were significantly increased for 14 days. In conclusion, these results suggested that $\mathrm{NF}-\kappa \mathrm{B}$ and ASIC3 may serve an important role in intervertebral disc degeneration caused by NP.
\end{abstract}

\section{Introduction}

Degeneration of the intervertebral disc is a part of the normal aging process, with the typical characteristic of disc extracellular matrix loss. Patients with intervertebral disc degeneration

Correspondence to: Dr Hao Pan, Department of Orthopaedics, The Affiliated Guang-Xing Hospital of Zhejiang TCM University, 453 Tiyuchang Road, Xihu, Hangzhou, Zhejiang 310007, P.R. China E-mail: haopanhz@126.com

Key words: intervertebral disc, nucleus pulposus, nuclear factor- $\kappa \mathrm{B}$, acid sensing ion channel 3, inflammation are often symptom free, despite large changes observed in the structure of discs; therefore, individuals with intervertebral disc degeneration who experience pain are categorized as having intervertebral disc disease (1). The mechanism of radicular pain due to disc herniation is associated with mechanical dorsal root ganglion (DRG) compression and inflammation, or autoimmune processes induced by application of nucleus pulposus (NP) onto the nerve root. Mechanical DRG compression induces long periods of repetitive firing (2), decrease in blood flow (3) and an increase in pain-associated behavior (4), as well as structural changes in the spinal cord (5). On the other hand, it has been reported that contact of NP with the nerve root causes a decrease in intraneural blood flow, as well as morphological and functional changes in the nerve root and DRG (6). It is not known, however, whether this sensitizing effect of NP on responses of nerve root reflects a central sensitization, is secondary to a peripheral sensitization, or a combination of both.

Previous research has provided evidence that intervertebral disc disease is associated with increased levels of pro-inflammatory cytokines in disc tissue produced by NP after injury, including tumor necrosis factor $\alpha$ (TNF- $\alpha)$, interleukin 6 (IL-6), IL-1 $\beta$ and IL-8 (7-9). Furthermore, immunoglobulins, hydrogen, nitric oxide (NO) and enzymes including phospholipase 2 are also responsible for the pathophysiological reactions in intervertebral disc disease (10). In summary, the inflammatory effects induced by the contact of the NP with nerve root are a complex event where numerous mediators and mechanisms serve a role at various levels.

Nuclear factor $(\mathrm{NF})-\kappa \mathrm{B}$ is a central component in the cellular response to damage, stress and inflammation, which have been implicated in the pathogenesis of many diseases, including musculoskeletal diseases such as osteoarthritis (11), osteoporosis (12), rheumatoid arthritis (13), and muscular dystrophy (14), but limited information of its role in intervertebral disc disease has been generated so far. Symptomatic discs are characterized by increases in level of TNF- $\alpha$, IL-1 $\beta$, IL-6 and IL- 8 , which are $\mathrm{NF}-\kappa \mathrm{B}$ target genes $(15,16)$, suggesting the involvement of $\mathrm{NF}-\kappa \mathrm{B}$ in intervertebral disc disease. Furthermore, activation of $\mathrm{NF}-\kappa \mathrm{B}$ signaling occurs in intervertebral disc degeneration, especially in the NP tissue, and was demonstrated to be associated with accumulated oxidative 
stress and matrix loss $(17,18)$. However, further investigation is needed to confirm if NF- $\mathrm{kB}$ is also involved in the development of a painful disc.

Acid sensing ion channel (ASIC) proteins are members of the amiloride-sensitive $\mathrm{Na}^{+}$-channel/degenerin family which form both homomeric and heteromeric functional membrane channels (19), of which ASIC3 has been implicated in pain transduction associated with ischemic or inflamed tissue acidosis (20), and is responsible for adaptation of the NP to the acidic and hyperosmotic microenvironment of the intervertebral disc (19).

To determine the mechanisms by which NF- $\mathrm{KB}$ and ASIC3 are involved in NP-induced intervertebral disc inflammatory process, the NF- $\kappa \mathrm{B}$ inhibitor pyrrolidine dithiocarbamate (PDTC) and the ASIC3 signaling pathway blocker amiloride were introduced, and the expression levels of inflammatory cytokines were also examined in an NP model.

\section{Materials and methods}

Animals. Male Sprague-Dawley rats (age, 6-8 weeks; weight, 220-240 g; n=80) were obtained from Shanghai Laboratory Animal Company (Shanghai, China). The animals were housed in temperature-controlled rooms $\left(22-25^{\circ} \mathrm{C}\right)$ with an alternating 12-h light/dark cycle. Water and food were available ad libitum. The experimental procedures were approved by the Guide for the Care and Use of Laboratory Animals of the National Institutes of Health (9th edition, 2010). The animal use protocol was approved by the Institutional Animal Care and Use Committee of Hangzhou Ding Qiao Hospital (Hangzhou, China).

Surgery. In all surgical procedures, the animals were anesthetized with an intraperitoneal injection of chloral hydrate (100 g/l; Shanghai Gushen Biological Technology Co., Ltd., Shanghai, China). The experimental model used in this study reproduced intervertebral disc degeneration caused by the implantation of autologous nucleus pulposus (NP) over the right L5-DRG. Briefly, a midline incision in the top of crista iliaca was bilaterally exposed, and the L5 inferior and L6 superior articular facet were removed. This procedure allowed for visualization of most of the dorsal side of the left L5 DRG. The NP $(0.4 \mathrm{mg})$ was removed through a transverse incision in the annulus fibrosus of the coccygeal disc and was placed over the L5-DRG. After this procedure, the surgical wound was sutured on a single plane with muscle fascia and skin. Animals were allocated to the following treatment groups: i) control group without treatment $(\mathrm{n}=16)$; ii) NP model group ( $\mathrm{n}=16$ ); iii) intraperitoneal injection of $20 \mu \mathrm{g} / \mathrm{kg}$ amiloride ( $\mathrm{n}=16)$; iv) intraperitoneal injection of $20 \mu \mathrm{g} / \mathrm{kg} \mathrm{NF}-\kappa \mathrm{B}$ inhibitor PDTC $(\mathrm{n}=16)$; and $\mathrm{v})$ intraperitoneal injection of both amiloride and PDTC $(n=16)$. The interventions described above were performed after the surgical procedures every day for 2 weeks. On completion of the experiment, the animal was sacrificed by anesthetized with $\mathrm{CO}_{2}$ and then a cervical dislocation was performed, and the DRG was collected.

Behavioral tests. Behavioral tests were performed to evaluate the decrease in the mechanical thresholds in the rats' hind paws. Briefly, mechanical hyperalgesia was measured by the electronic von Frey method. A total of 30 min before the beginning of the tests, the rats were placed in acrylic cages with wire grid floors. The stimulus was automatically discontinued and its intensity recorded when the paw was withdrawn. The stimulus was repeated 3 times at 5-min intervals until the animal presented similar measurements. The animals were tested 2, 4, 6, 8, 10 and 12 days after the surgical procedures.

$R N A$ extraction and reverse transcription-quantitative polymerase chain reaction ( $R T-q P C R)$. Total RNA was extracted using TRIzol reagent (Gibco; Thermo Fisher Scientific, Inc., Waltham, MA, USA) according to manufacturer's protocol. RNA $(1 \mu \mathrm{g})$ was reverse transcribed to cDNA using a cDNA synthesis kit (Thermo Fisher Scientific, Inc.). qPCR was performed to measure the mRNA expression levels and the data was analyzed using an ABI-7300 system (Applied Biosystems; Thermo Fisher Scientific, Inc.). Maxima SYBR Green/ROX qPCR Master Mix (cat. no. K0223; Finnzymes; Thermo Fisher Scientific, Inc.) was used, according to the manufacturer's protocol. The gene expression was calculated using the $2-\Delta \Delta \mathrm{Cq}$ method (21). Primers were as follows: NF-kBp65, forward, 5'-TTTAGCCTGCTGGCGGTTCC-3' and reverse, 5'-ACCGAGTGCAGCCGTGATTG-3'; ASIC3, forward, 5'-CGCTATGTGGCTCGGAAGTG-3' and reverse, 5'-TGTAGGACTCGCTGCGGTTG-3'; aquaporin (AQP) 1, forward, 5'-AGAGCCTGGACAATCTGAAG-3' and reverse, 5'-TTAACGGCACAGTGGTAGAG-3'; AQP3, forward, 5'-CTTCTTGGGTGCTGGGATTG-3' and reverse, 5'-GCT GCTGTGCCTATGAACTG-3'; TNF- $\alpha$, forward, 5'-CTG AGGTCAACCTGCCCAAG-3' and reverse, 5'-GGCTGG GTAGAGAACGGATG-3'; IL-6, forward, 5'-CACCAGGAA CGAAGTCAAC-3' and reverse, 5'-GGCTGTCAACAACAT CAGTC-3'; GAPDH, forward, 5'-GTCGGTGTGAACGGA TTTG-3' and reverse, 5'-TCCCATTCTCAGCCTTGAC-3'. The PCR cycling conditions were as follows: $95^{\circ} \mathrm{C}$ for $10 \mathrm{~min}$, followed by 40 cycles at $95^{\circ} \mathrm{C}$ for $15 \mathrm{sec}$ and $60^{\circ} \mathrm{C}$ for $45 \mathrm{sec}$, and a final extension step of $95^{\circ} \mathrm{C}$ for $15 \mathrm{sec}, 60^{\circ} \mathrm{C}$ for $1 \mathrm{~min}$, $95^{\circ} \mathrm{C}$ for $15 \mathrm{sec}$ and $60^{\circ} \mathrm{C}$ for $15 \mathrm{sec}$.

Protein extraction and western blotting. Protein was extracted from the DRG and an intervertebral disc using radioimmuno precipitation buffer (JRDUN Biotechnology Co., Ltd., Shanghai, China). Protein lysates $(30 \mu \mathrm{g})$ were separated by 10-15\% SDS-PAGE (Sigma-Aldrich; Merck KGaA, Darmstadt, Germany) electrophoresis using a Bio-Rad miniature slab gel apparatus (Bio-Rad Laboratories, Inc., Hercules, CA, USA) and proteins were electrophoretically transferred onto a nitrocellulose membrane (EMD Millipore, Billerica, MA, USA). The membrane was first incubated with rabbit monoclonal primary antibodies against NF-кBp65 (cat. no. 6956; 1:1,000; Cell Signaling Technology, Inc., Danvers, MA, USA), ASIC3 (cat. no. ab49333; 1:400; Abcam, Cambridge, MA, USA), AQP1 (cat. no. ab65837; 1:500; Abcam), AQP3 (cat. no. sc-20811; 1:500; Santa Cruz Biotechnology, Dallas, TX, USA), TNF- $\alpha$ (cat. no. ab6671; 1:1,000; Abcam) and a mouse polyclonal antibody against IL-6 (cat. no. ab9324; 1:1,000; Abcam) overnight at $4^{\circ} \mathrm{C}$, followed by an anti-GAPDH (cat. no. 5174; 1:1,500; Cell Signaling Technology, Inc.) antibody as a loading control. Subsequently, the membrane was incubated with a horseradish 
A

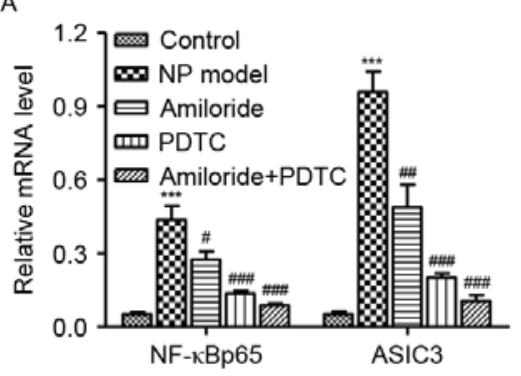

B

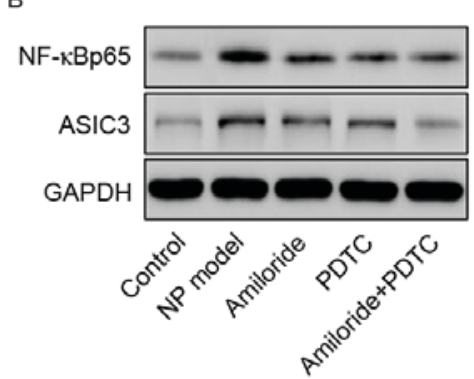

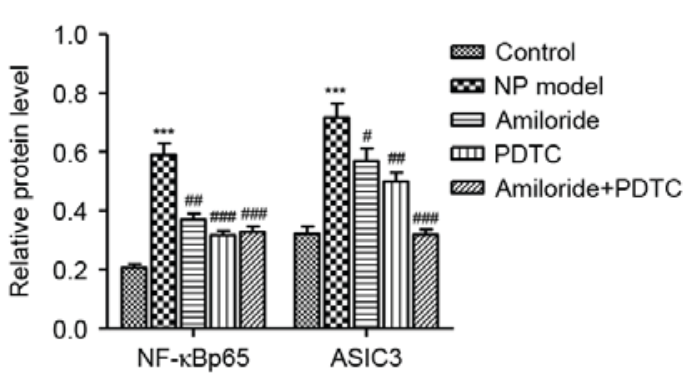

Figure 1. Determination of NF- $\mathrm{B}$ and ASIC3 expression levels in the DRG of rats. At 14 days after surgery, NF- $\kappa$ Bp65 and ASIC3 expression was measured by (A) reverse transcription-quantitative polymerase chain reaction and (B) western blotting. Data are presented as the mean \pm standard deviation. ${ }^{* * *} \mathrm{P}<0.001$ vs. control. ${ }^{\#} \mathrm{P}<0.05,{ }^{\# \#} \mathrm{P}<0.01,{ }^{\# \#} \mathrm{P}<0.001$ vs. NP model. NP, nucleus pulposus; PDTC, pyrrolidine dithiocarbamate; NF- $\kappa \mathrm{B}$, nuclear factor- $\kappa \mathrm{B}$; ASIC3, acid sensing ion channel 3 .

peroxidase-conjugated secondary antibody (Beyotime Institute of Biotechnology, Haimen, China; cat. nos. A0208 and A0216; $1: 1,000)$ for $1 \mathrm{~h}$ at $37^{\circ} \mathrm{C}$, prior to being washed three times with Tris-buffered saline with 20\% Tween-20 (Amresco, LLC, Solon, OH, USA). The blots were visualized using enhanced chemiluminescence (EMD Millipore) and the signal intensity was determined using Image J software version 1.46 (National Institutes of Health, Bethesda, MD, USA).

ELISA. TNF- $\alpha$ and IL-6 concentrations present in peripheral blood and DRG were determined 1, 3, 7 and 14 days after the surgical procedures using commercially available murine-specific sandwich ELISA kits (cat. nos. RTA00 and R6000B, respectively; R\&D Systems, Inc., Minneapolis, MN, USA), following the manufacturer's protocol.

Measurement of nitric oxide (NO). Peripheral blood and DRG were centrifuged at $1,000 \times \mathrm{g}$ for $10 \mathrm{~min}$ at $4^{\circ} \mathrm{C}$, and the supernatant was collected to determine the concentration of NO 1, 3, 7 and 14 days after the surgical procedures using an NO assay kit (Nanjing Jiancheng Bioengineering Institute, Nanjing, China).

Statistical analysis. The data are presented as the mean \pm standard deviation. Statistical analyses were performed using GraphPad Prism software, version 5 (GraphPad Software, Inc., La Jolla, CA, USA). Data were analysed by one-way analysis of variance followed by Tukey's post hoc test. $\mathrm{P}<0.05$ was considered to indicate a statistically significant difference.

\section{Results}

Expression of $N F-\kappa B$ and ASIC3 in rat DRG. A total of 14 days after surgery, the mRNA and protein expression levels of NF- $\kappa$ B and ASIC3 were measured in the DRG by RT-qPCR and western blotting, respectively. The mRNA and protein expression levels of $\mathrm{NF}-\kappa \mathrm{Bp} 65$ and ASIC3 were significantly increased compared with the control groups (Fig. 1A and B, respectively). However, a significant decrease in the mRNA and protein expression levels of NF- $\kappa$ Bp65 and ASIC3 was observed in amiloride or/and PDTC group compared with the NP model group (Fig. 1A and B). These data indicated that amiloride and PDTC could inhibit the activation of NF- $\kappa$ Bp65 and ASIC3 in DRG induced by NP treatment.

Behavioral testing. A significant difference in mechanical pain withdrawal threshold (PWT) between the NP model and the control groups were observed from days 2 to 12 after surgery (Fig. 2, P<0.001). The mechanical PWT decreased for 8 days after surgery and then gradually increased in the amiloride and PDTC groups. Furthermore, a significant increase in PWT in the amiloride and PDTC groups compared with the NP model groups were observed at days 10 and 12 after surgery, respectively (Fig. 2, P<0.001). In the amiloride plus PDTC groups, a significant increase was observed from day 8 to 12 after surgery compared with the amiloride or PDTC treatment alone group $(\mathrm{P}<0.05$ and $\mathrm{P}<0.01$, respectively). These results suggested that amiloride and PDTC could reduce mechanical hyperalgesia induced by NP treatment.

Expression of NO, TNF- $\alpha$ and IL- 6 in the peripheral blood and DRG. At 1, 3, 7 and 14 days after surgery, the concentration of NO in the peripheral blood and DRG was measured using ELISA and biochemical assays. As presented in Fig. 3A and $\mathrm{B}(\mathrm{P}<0.001)$, the concentrations of $\mathrm{NO}$ were significantly increased from days 1 to 14 in peripheral blood and DRG of the rats with NP treatment compared with the control groups. However, amiloride or/and PDTC treatment significantly inhibited the increases in NO concentration induced by NP treatment from days 3 to 14 (Fig. $3 \mathrm{~A}$ and $\mathrm{B}, \mathrm{P}<0.01, \mathrm{P}<0.001$ vs. NP model).

Subsequently, concentrations of the inflammatory factors TNF- $\alpha$ and IL- 6 in peripheral blood and DRG were also examined 1, 3, 7 and 14 days after surgery by ELISA. ELISA assay demonstrated that the concentration of TNF- $\alpha$ and IL-6 was significantly increased by NP treatment compared with the control groups (Fig. 3C-F, $\mathrm{P}<0.001$ ). However, amiloride or/and PDTC treatment significantly inhibited the increases in TNF- $\alpha$ and IL- 6 concentration induced by NP treatment from days 3 to 14 (Fig. 3C-F, $\mathrm{P}<0.01, \mathrm{P}<0.001$ vs. NP model). Similar results were also observed 14 days after surgery in the peripheral blood and DRG, as assessed by RT-qPCR and western blotting (Fig. 3G and $\mathrm{H}$, respectively). These data indicated that amiloride and PDTC could inhibit the secretion of NO, TNF- $\alpha$ and IL- 6 in peripheral blood and DRG induced by NP treatment. 


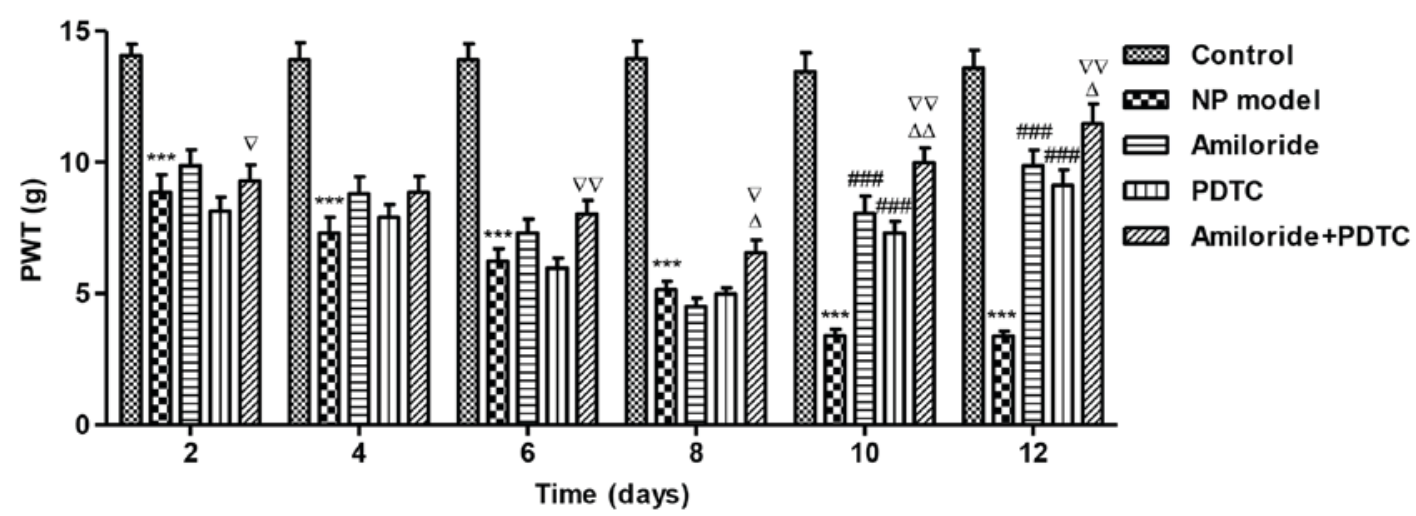

Figure 2. Changes in mechanical PWT in rats. Mechanical PWT was measured by the electronic von Frey method. Data are presented as the mean \pm standard deviation. ${ }^{* * *} \mathrm{P}<0.001$ vs. control. ${ }^{\# \# \#} \mathrm{P}<0.001$ vs. NP model. ${ }^{\triangle} \mathrm{P}<0.05,{ }^{\triangle} \triangle \mathrm{P}<0.01$ vs. amiloride. ${ }^{\nabla} \mathrm{P}<0.05,{ }^{\nabla} \mathrm{P}<0.01$ vs. PDTC. PWT, pain withdrawal threshold; NP, nucleus pulposus; PDTC, pyrrolidine dithiocarbamate.
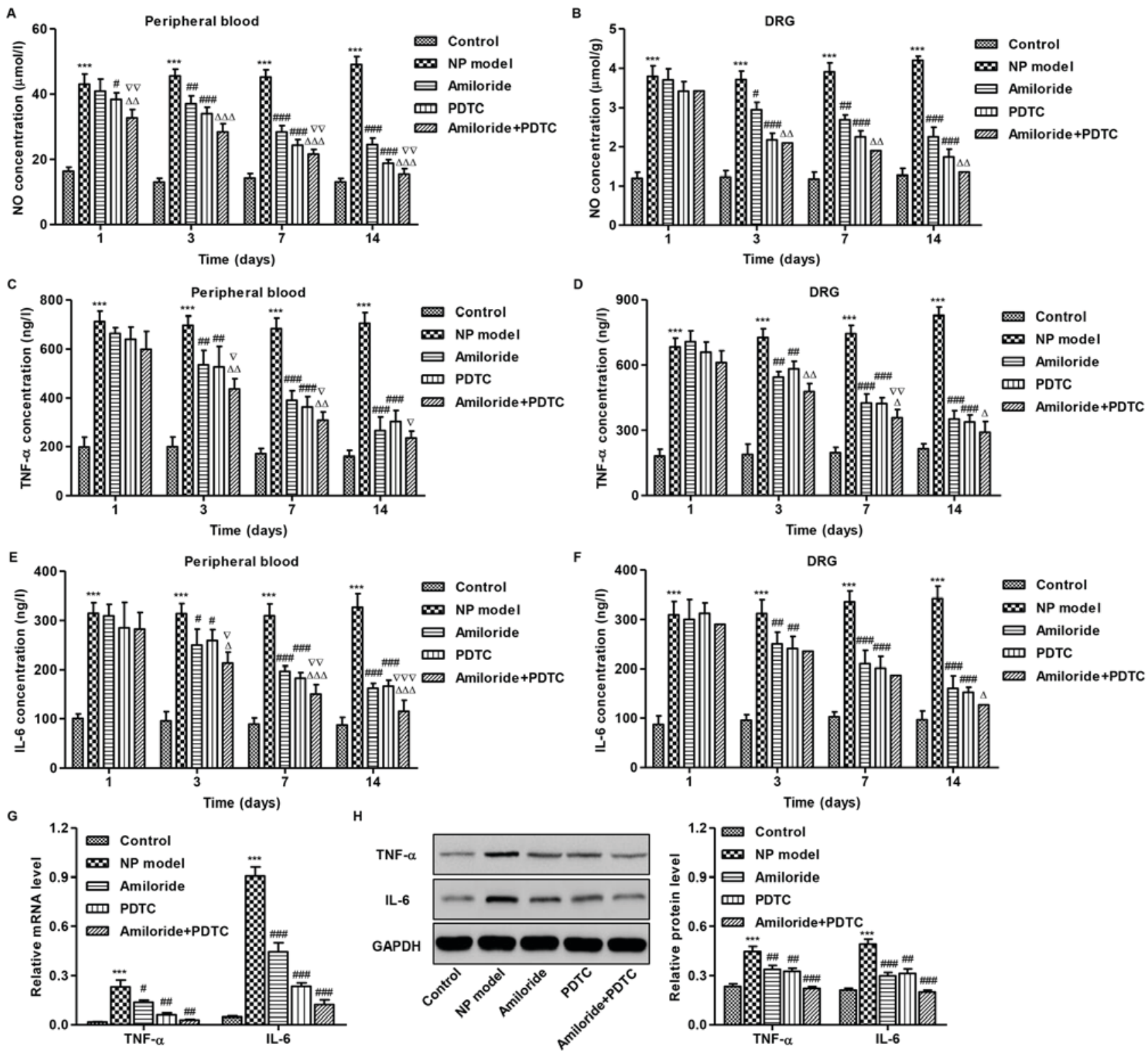

Figure 3. Determination of NO, TNF- $\alpha$ and IL-6 levels in the peripheral blood and DRG of rats. At 1, 3, 7 and 14 days after surgery, NO concentration in peripheral blood and DRG was measured by (A) ELISA and (B) biochemical assay. ELISA measurements of TNF- $\alpha$ concentration in (C) peripheral blood and (D) DRG; and IL-6 concentration in (E) peripheral blood and (F) DRG. At 14 days after surgery, TNF- $\alpha$ and IL-6 expression in the DRG was measured by (G) reverse transcription-quantitative polymerase chain reaction and $(\mathrm{H})$ western blotting. Data are presented as the mean \pm standard deviation. ${ }^{* * *} \mathrm{P}<0.001$ vs. control. ${ }^{\# \mathrm{P}}<0.05,{ }^{\# \#} \mathrm{P}<0.01,{ }^{\# \# \#} \mathrm{P}<0.001$ vs. NP model. ${ }^{\triangle} \mathrm{P}<0.05,{ }^{\triangle} \mathrm{P}<0.01,{ }^{\Delta} \Delta \triangle \mathrm{P}<0.001$ vs. amiloride. ${ }^{\nabla} \mathrm{P}<0.05,{ }^{\nabla \nabla} \mathrm{P}<0.01,{ }^{\nabla \nabla} \mathrm{P}<0.001$ vs. PDTC. NO, nitric oxide; DRG, dorsal root ganglion; TNF- $\alpha$, tumor necrosis factor- $\alpha$; IL-6, interleukin-6. 

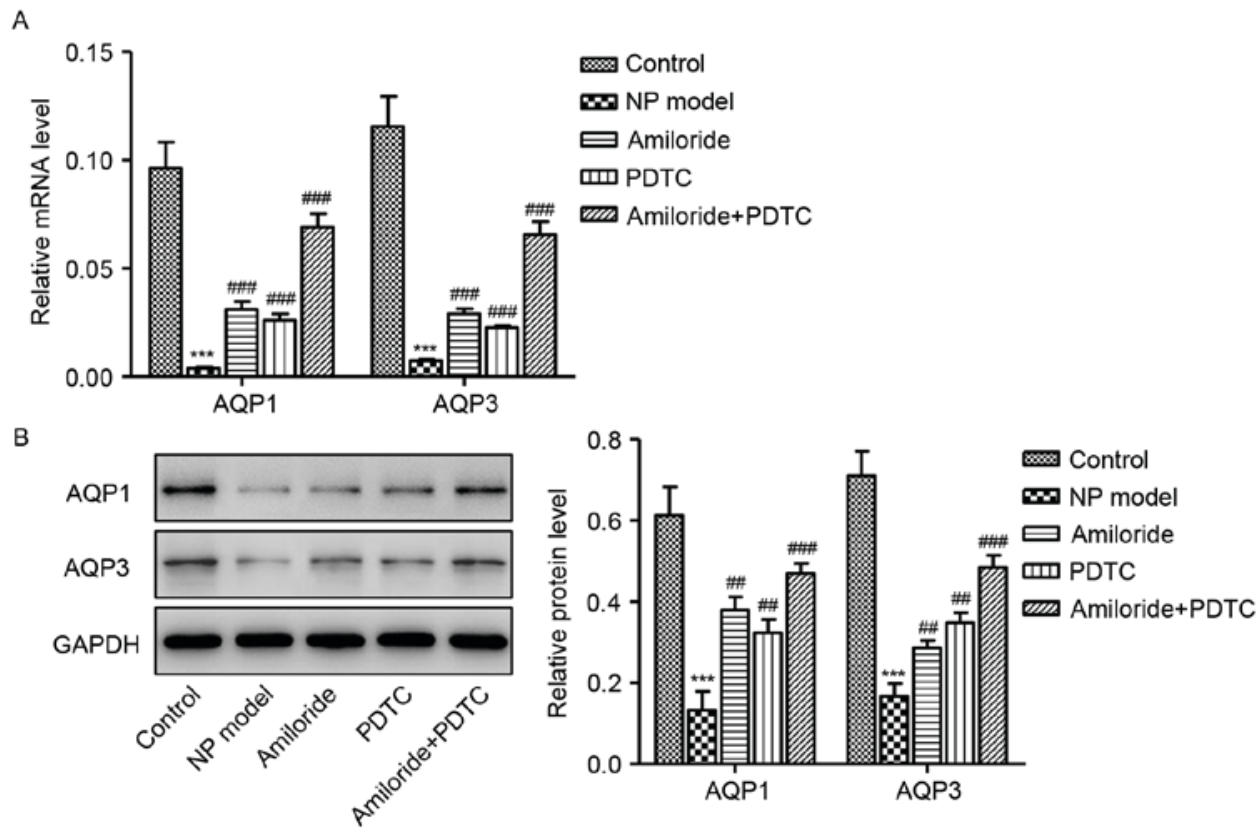

Figure 4. Determination of AQP1 and AQP3 expression levels in the intervertebral disc of rats. At 14 days after surgery, AQP1 and AQP3 mRNA and protein expression levels were measured by (A) reverse transcription-quantitative polymerase chain reaction and (B) western blotting, respectively. Data are presented as the mean \pm standard deviation. ${ }^{* * *} \mathrm{P}<0.001$ vs. control. ${ }^{\#} \mathrm{P}<0.01,{ }^{\# \#} \mathrm{P}<0.001$ vs. NP model. AQP, aquaporin; NP, nucleus pulposus; PDTC, pyrrolidine dithiocarbamate.

Expression of $A Q P 1$ and $A Q P 3$ in the intervertebral disc. A total of 14 days after surgery, the expression of AQP1 and AQP3 in the intervertebral disc was measured by RT-qPCR and western blotting. It was observed that the mRNA (Fig. 4A) and protein (Fig. 4B) expression levels of AQP1 and AQP3 significantly decreased in the NP model group compared with the control groups. However, a significant increase in the mRNA and protein expression levels of AQP1 and AQP3 was observed in amiloride or/and PDTC groups compared with the NP model group. These data indicated that amiloride and PDTC could ameliorate the expression levels of AQP1 and AQP3 in the intervertebral disc induced by NP treatment.

\section{Discussion}

The intervertebral disc is a specialized biomechanical structure containing NP that secretes matrix macromolecules, which by elevating the osmotic pressure, serves to accommodate applied mechanical forces. Many recent studies have shifted the focus toward potential involvement of inflammatory processes initiated by contact of NP with the nerve root and surrounding tissues $(10,22)$. As the NP can be regarded as a closed tissue compartment with a limited vascular supply, the present study explored the possibility that ASIC3 expression is increased by NP application on the DRG compared with control DRG without NP treatment, suggesting that NP could simulate the expression of ASIC3 in DRG. The present study demonstrated that the level of $\mathrm{NF}-\kappa \mathrm{B}$ was enhanced after 14 days in an NP-induced intervertebral disc degeneration rat model, in line with previous studies $(17,18)$. However, intraperitoneal injection of $20 \mu \mathrm{g} / \mathrm{kg}$ amiloride or/and PDTC markedly inhibited the increases of ASIC 3 and NF- $\kappa$ B expression in DRG induced by the contact of NP with the nerve root.
Hyperalgesia is a common denominator of all inflammatory processes and is characterized by a decrease in the nociceptive threshold, and increased activity in response to thermal and mechanical stimulation (23). In the present study, reduction in the mechanical PWT by NP induction was marked in the 12 days after surgery, with a significant fall starting on day 10. This was consistent with our previous study, in which disc herniation experimental model rats, in which NP is deposited over the DRG, demonstrated increased mechanical hyperalgesia that lasted $>7$ weeks (10). However, in the present study the hyperalgesia induced by NP was significantly improved in rats following amiloride and PDTC treatment, with a significant rise starting on day 8 after surgery, indicating that ASIC3 and NF- $\kappa \mathrm{B}$ may be involved in NP-induced nerve root injury.

Numerous cytokines such as TNF- $\alpha$ and IL-6 are associated with increased neuropeptides, and the results observed with cytokine inhibition in previous studies suggested that blocking induction upstream of several cytokines is desirable for blocking the sensation of pain both in the peripheral and central nervous systems $(24,25)$. In the present study, NO, TNF- $\alpha$ and IL- 6 levels were increased both in peripheral blood and DRG 14 days after NP treatment. Furthermore, the protein expression levels of NO, TNF- $\alpha$ and IL- 6 in DRG were measured by western blotting, and the results were consistent with those mentioned above. However, amiloride or/and PDTC markedly reduced the increases in NO, TNF- $\alpha$ and IL-6 concentration in peripheral blood and DRG from days 3 to 14 after NP treatment.

It has been hypothesized that loss of AQPs may be the cause of degeneration of the intervertebral disc. In the present study, the expression of AQP1 and AQP3 was significantly decreased in NP-simulated intervertebral discs, while amiloride or/and PDTC corrected the effects of NP on AQP1 
and AQP3 expression. AQP1 and AQP3 have previously been demonstrated to have important roles in degeneration of the intervertebral disc $(26,27)$. Xie et al (28) demonstrated that AQP3 protects against lumbar intervertebral disc degeneration via the $\mathrm{Wnt} / \beta$-catenin signaling pathway. Although previous studies have indicated the altered expression pattern of AQP1 and AQP3 in human intervertebral disc, to the best of our knowledge, this study is the first to compare the expression of AQP1 and AQP3 in NP-simulated intervertebral discs of rats.

In the present study, the expression of ASIC3 and NF- $\mathrm{kB}$ was examined in NP-simulated DRG of rats. Further functional studies demonstrated that inhibition of ASIC3 and $\mathrm{NF}-\kappa \mathrm{B}$ could suppress the effects of NP on DRG injury, evidenced by decreases in $\mathrm{NO}$ and inflammatory cytokine (TNF- $\alpha$ and IL-6) concentration, as well as increases in AQP1 and AQP3 expression. These data suggested that ASIC3 and $\mathrm{NF}-\kappa \mathrm{B}$ might have significant roles for the development of intervertebral disc degeneration, and may represent novel therapeutic targets.

\section{Acknowledgements}

The present study was supported by Hang Zhou Science and Technology Funds (grant no. 20150733Q60) and Zhejiang Province Traditional Chinese Medicine Science and Technology Planning Project (grant no. 2015ZA157).

\section{References}

1. Wuertz K, Vo N, Kletsas D and Boos N: Inflammatory and catabolic signalling in intervertebral discs: The roles of NF- $\mathrm{KB}$ and MAP kinases. Eur Cell Mater 23: 103-120, 2012.

2. Tan ZY, Donnelly DF and LaMotte RH: Effects of a chronic compression of the dorsal root ganglion on voltage-gated $\mathrm{Na}+$ and $\mathrm{K}+$ currents in cutaneous afferent neurons. J Neurophysiol 95: $1115-1123,2006$.

3. Igarashi T, Yabuki S, Kikuchi S and Myers RR: Effect of acute nerve root compression on endoneurial fluid pressure and blood flow in rat dorsal root ganglia. J Orthop Res 23: 420-424, 2005.

4. Ma C and LaMotte RH: Enhanced excitability of dissociated primary sensory neurons after chronic compression of the dorsal root ganglion in the rat. Pain 113: 106-112, 2005.

5. Watanabe K, Konno S, Sekiguchi M, Sasaki N, Honda T and Kikuchi S: Increase of 200-kDa neurofilament-immunoreactive afferents in the substantia gelatinosa in allodynic rats induced by compression of the dorsal root ganglion. Spine (Phila $\mathrm{Pa}$ 1976) 32: 1265-1271, 2007.

6. Miyoshi S, Sekiguchi M, Konno S, Kikuchi S and Kanaya F: Increased expression of vascular endothelial growth factor protein in dorsal root ganglion exposed to nucleus pulposus on the nerve root in rats. Spine (Phila Pa 1976) 36: E1-E6, 2011.

7. Sasaki N, Sekiguchi M, Kikuchi S and Konno S: Effects of asialo-erythropoietin on pain-related behavior and expression of phosphorylated-p38 map kinase and tumor necrosis factor-alpha induced by application of autologous nucleus pulposus on nerve root in rat. Spine (Phila Pa 1976) 36: E86-E94, 2011.

8. Le Maitre CL, Hoyland JA and Freemont AJ: Catabolic cytokine expression in degenerate and herniated human intervertebral discs: IL-1beta and TNFalpha expression profile. Arthritis Res Ther 9: R77, 2007.

9. Shamji MF, Setton LA, Jarvis W, So S, Chen J, Jing L, Bullock R, Isaacs RE, Brown C and Richardson WJ: Proinflammatory cytokine expression profile in degenerated and herniated human intervertebral disc tissues. Arthritis Rheum 62: 1974-1982, 2010.
10. de Souza Grava AL, Ferrari LF and Defino HL: Cytokine inhibition and time-related influence of inflammatory stimuli on the hyperalgesia induced by the nucleus pulposus. Eur Spine J 21: 537-545, 2012.

11. Imagawa $\mathrm{K}$, de Andrés MC, Hashimoto K, Pitt D, Itoi E, Goldring MB, Roach HI and Oreffo RO: The epigenetic effect of glucosamine and a nuclear factor-kappa B (NF- $\mathrm{B}$ ) inhibitor on primary human chondrocytes-implications for osteoarthritis. Biochem Biophys Res Commun 405: 362-367, 2011.

12. Hein GE: Glycation endproducts in osteoporosis-is there a pathophysiologic importance? Clin Chim Acta 371: 32-36, 2006.

13. Roman-Blas JA and Jimenez SA: NF-kappaB as a potential therapeutic target in osteoarthritis and rheumatoid arthritis. Osteoarthritis Cartilage 14: 839-848, 2006.

14. Messina S, Vita GL, Aguennouz M, Sframeli M, Romeo S, Rodolico C and Vita G: Activation of NF-kappaB pathway in Duchenne muscular dystrophy: Relation to age. Acta Myol 30: 16-23, 2011.

15. Adams MA, Stefanakis M and Dolan P: Healing of a painful intervertebral disc should not be confused with reversing disc degeneration: Implications for physical therapies for discogenic back pain. Clin Biomech (Bristol, Avon) 25: 961-971, 2010.

16. Hoyland JA, Le Maitre $C$ and Freemont AJ: Investigation of the role of IL-1 and TNF in matrix degradation in the intervertebral disc. Rheumatology (Oxford) 47: 809-814, 2008.

17. Nerlich AG, Bachmeier BE, Schleicher E, Rohrbach H, Paesold G and Boos N: Immunomorphological analysis of RAGE receptor expression and NF-kappaB activation in tissue samples from normal and degenerated intervertebral discs of various ages. Ann N Y Acad Sci 1096: 239-248, 2007.

18. Akeda K, An H, Gemba T, Okuma M, Miyamoto K, Chujo T, Kitahara S and Masuda K: A new gene therapy approach: In vivo transfection of naked NFkB decoy oligonucleotide restored disc degeneration in the rabbit annular needle puncture model. Trans Orthop Res Soc 30: 45, 2005.

19. Uchiyama Y, Cheng CC, Danielson KG, Mochida J, Albert TJ, Shapiro IM and Risbud MV: Expression of acid-sensing ion channel 3 (ASIC3) in nucleus pulposus cells of the intervertebral disc is regulated by p75NTR and ERK signaling. $\mathrm{J}$ Bone Miner Res 22: 1996-2006, 2007.

20. Yagi J, Wenk HN, Naves LA and McCleskey EW: Sustained currents through ASIC 3 ion channels at the modest $\mathrm{pH}$ changes that occur during myocardial ischemia. Circ Res 99: 501-509, 2006.

21. Livak KJ and Schmittgen TD: Analysis of relative gene expression data using real-time quantitative PCR and the 2(-Delta Delta C(T)) method. Methods 25: 402-408, 2001.

22. Ohtori S, Inoue G, Eguchi Y, Orita S, Takaso M, Ochiai N, Kishida S, Kuniyoshi K, Aoki Y, Nakamura J, et al: Tumor necrosis factor- $\alpha$-immunoreactive cells in nucleus pulposus in adolescent patients with lumbar disc herniation. Spine (Phila Pa 1976) 38: 459-462, 2013.

23. Joseph EK, Green PG, Bogen O,Alvarez P and Levine JD: Vascular endothelial cells mediate mechanical stimulation-induced enhancement of endothelin hyperalgesia via activation of $\mathrm{P} 2 \mathrm{X} 2 / 3$ receptors on nociceptors. J Neurosci 33: 2849-2859, 2013.

24. Studer RK, Aboka AM, Gilbertson LG, Georgescu H, Sowa G, Vo N and Kang JD: p38 MAPK inhibition in nucleus pulposus cells: A potential target for treating intervertebral disc degeneration. Spine (Phila Pa 1976) 32: 2827-2833, 2007.

25. Hayashi Y, Ohtori S, Yamashita M, Yamauchi K, Inoue G, Suzuki M, Orita S, Eguchi Y, Ochiai N, Kishida S, et al: Direct single injection of p38 mitogen-activated protein kinase inhibitor does not affect calcitonin gene-related peptide expression in dorsal root ganglion neurons innervating punctured discs in rats. Spine (Phila Pa 1976) 34: 2843-2847, 2009.

26. Li SB, Yang KS and Zhang YT: Expression of aquaporins 1 and 3 in degenerative tissue of the lumbar intervertebral disc. Genet Mol Res 13: 8225-8233, 2014.

27. Tas U, Cayli S, Inanir A, Ozyurt B, Ocaklı S, Karaca Zİ and Sarsilmaz M: Aquaporin-1 and aquaporin-3 expressions in the intervertebral disc of rats with aging. Balkan Med J 29: 349-353, 2012.

28. Xie H, Jing Y, Xia J, Wang X, You C and Yan J: Aquaporin 3 protects against lumbar intervertebral disc degeneration via the Wnt/ß-catenin pathway. Int J Mol Med 37: 859-864, 2016. 